Journal of Biomechanics, in press.

\title{
A kinematic algorithm to identify gait events during running at different speeds and with different footstrike types
}

\author{
1,2Joe C Handsaker, ${ }^{3}$ Stephanie E Forrester, ${ }^{1}$ Jonathan P Folland, ${ }^{1}$ Matt I Black, and \\ ${ }^{1}$ Sam J Allen.
}

\begin{abstract}
${ }^{1}$ School of Sport, Exercise and Health Sciences, Loughborough University, Leicestershire, LE11 3TU, UK
${ }^{2}$ MAS Innovation, Colombo, Sri Lanka

${ }^{3}$ Wolfson School of Mechanical, Electrical and Manufacturing Engineering, Loughborough University, Leicestershire, LE11 3TU, UK
\end{abstract}

\begin{abstract}
Although a number of algorithms exist for estimating ground contact events (GCEs) from kinematic data during running, they are typically only applicable to heelstrike running, or have only been evaluated at a single running speed. The purpose of this study was to investigate the accuracy of four kinematics-based algorithms to estimate GCEs over a range of running speeds and footstrike types. Subjects ran over a force platform at a range of speeds; kinetic and kinematic data was captured at $1000 \mathrm{~Hz}$, and kinematic data was downsampled to $250 \mathrm{~Hz}$. A windowing process initially identified reduced time windows containing touchdown and toe-off. Algorithms based on acceleration and jerk signals of the foot markers were used to estimate touchdown (2 algorithms), toe-off (2 algorithms), and ground contact time (GCT) (4 algorithms), and compared to synchronous 'gold standard' force platform data. An algorithm utilising the vertical acceleration peak of either the heel or first metatarsal marker (whichever appeared first) for touchdown, and the vertical jerk peak of the hallux marker for toe-off, resulted in the lowest offsets $(+3.1 \mathrm{~ms}, 95 \%$ Confidence Interval $(\mathrm{Cl}):-11.8$ to $+18.1 \mathrm{~ms}$; and $+2.1 \mathrm{~ms}$, $\mathrm{Cl}:-8.1$ to $+12.2 \mathrm{~ms}$ respectively). This method also resulted in the smallest offset in GCT $(-1.1 \mathrm{~ms}, \mathrm{Cl}$ : -18.6 to $+16.4 \mathrm{~ms})$. Offsets in GCE and GCT estimates from all algorithms were typically negatively correlated to running speed, with offsets decreasing as speed increased. Assessing GCEs and GCT using this method may be useful when a force platform is unavailable or impractical.
\end{abstract}

Keywords: running, ground contact time, touchdown, toe-off

\section{INTRODUCTION}

The identification of ground contact events (GCEs) is an important aspect of gait analysis, allowing for the partitioning of motion capture recordings into gait cycles, and for comparisons to be made between individuals and gait cycles at discrete points. Although force data remains the current gold standard (Hansen et al., 2002), in many circumstances it is not possible to use a force platform, e.g. during field-based or standard treadmill analyses. Consequently, a number of kinematics-based algorithms to detect GCEs have been developed. Several authors have proposed algorithms for walking (De Witt et al., 2010; O'Connor et al., 2007; Zeni et al., 2008), and for running using only foot-based markers (Leitch et al. 2011; Maiwald et al., 2009), only markers on more proximal segments (Milner et al., 2015), as well as both foot-based and more proximal segment markers (Fellin et al., 2010; Hreljac \& Stergiou, 2000; Smith et al. 2015). One algorithm has been proposed for both walking and running, although the running speeds were relatively low $\left(2.5-3.6 \mathrm{~m} \cdot \mathrm{s}^{-1}\right)$ (Alvim et al., 2015). Existing running algorithms have typically been limited to either only rearfoot strike running (Alvim et al., 2015; Hreljac \& Stergiou, 2000) and / or single speed or multiple slow running speeds (Alvim et al., 2015; Fellin et al., 2010; Milner et al., 2015; Smith et al., 2015) and few have reported the accuracy of ground contact time (GCT) as well as the individual touchdown and toe-off GCEs (Hreljac \& Stergiou, 2000; Smith et al., 2015). 
The purpose of this study was to investigate the accuracy of four kinematics-based algorithms to estimate GCEs over a range of speeds and footstrike types. Two algorithms were used to estimate touchdown and two to estimate toe-off, which, when combined, gave four estimates of GCT.

\section{METHODOLOGY}

\subsection{Participants and protocol}

Twenty young, healthy, and physically active subjects (males: $n=12$, age 30.0 years \pm 5.5 years, height $1.81 \mathrm{~m} \pm 0.08 \mathrm{~m}$, mass $85.6 \mathrm{~kg} \pm 20.5 \mathrm{~kg}$; females: $n=8$, age 30.3 years \pm 7.4 years, height $1.64 \mathrm{~m} \pm 0.03 \mathrm{~m}$, mass $61.9 \mathrm{~kg} \pm 8.4 \mathrm{~kg}$ ) provided voluntary informed consent to participate in a protocol which was approved by the Loughborough University Ethics Committee. Participants were required to have been free from musculoskeletal injury in the three months prior to testing.

Participants wore a neutral racing flat running shoe (New Balance RC 1400 v2) with retro-reflective markers placed over the posterior aspect of the calcaneus (Heel), the superior aspect of the $1^{\text {st }}$ metatarsal head (Met) and the distal end of the hallux (Toe) on both shoes. A ten camera motion capture system (Vicon Nexus, Oxford Metrics Ltd, UK) and force platform (AMTI BP600400; Watertown, MA, USA) synchronously sampling at $1000 \mathrm{~Hz}$, recorded participants as they ran over the force platform at three self-selected speeds (slow, intermediate, and fast). Five 'good' trials per foot were recorded at each speed, giving a total of 30 trials per participant. A good trial was defined as one in which the foot contacted the centre of the force platform with minimal targeting.

\subsection{Data analysis}

The raw kinematic data was labelled, low-pass filtered at $15 \mathrm{~Hz}$ using a zerophase fourth order Butterworth filter, and downsampled to $250 \mathrm{~Hz}$ using Vicon software. The data was exported to Visual 3D (v5.01, C-Motion, Germantown, MD, USA) where vertical and horizontal (anterior-posterior) velocity, acceleration, and the first derivative of acceleration with respect to time (jerk) of each marker was calculated. Onset and cessation of vertical force was used as the gold standard for the identification of touchdown (TDF), toe-off (TOF), and ground contact time (GCTF) based on a threshold of $5 \mathrm{~N}$ (Figure 1a). Each trial was defined as a rearfoot strike (RFS), midfoot strike (MFS), or forefoot strike (FFS) based on footstrike angle (Altman and Davis, 2012).

All kinematic algorithms required touchdown and toe-off windows to be defined to limit the search regions. These windowing conditions were selected through a combination of visual observation of the foot marker displacement and velocity time profiles and a consideration of the foot movements involved in a typical running footstrike. The touchdown window started when the anterior-posterior velocity of the Heel first dropped below $1.5 \mathrm{~m} \cdot \mathrm{s}^{-1}$ and ended at the next minima in vertical Heel position. The toe-off window started $100 \mathrm{~ms}$ after touchdown, and ended when the vertical Toe position either exceeded $0.1 \mathrm{~m}$ or reached a maximum (Figure 1b). Notably, these cut-off values were able to successfully define TD and TO windows across the full range of ground contact times and, by implication, running speeds in this study.

Touchdown identification was based on the peak vertical acceleration or jerk of the Heel and Met markers. For RFS running where the heel impacts the ground first, the Heel peak would be expected to coincide with touchdown, while for FFS running the Met peak would be expected to better coincide with touchdown. Using the first 
occurring of these two peaks allows a range of footstrike types to be automatically accounted for within the algorithm. Toe-off identification was based on the peak vertical acceleration or jerk of the Toe, selected since the toe is the last point of contact with the ground irrespective of footstrike. It is expected that the timing of these peaks with respect to TD and TO remain relatively consistent across running speeds, the major effect of increasing speed being to increase the magnitude of the peaks.

The first touchdown algorithm was an adapted version of that proposed in a number of previous studies (Alvim et al., 2015; Leitch et al. 2011; Maiwald et al., 2009) and involved identifying the vertical acceleration peaks of the Heel and Met markers within the touchdown window. The instant of touchdown was then defined by the first of these peaks $\left(T D_{A}\right)$. It was expected that for a RFS the Heel peak would occur first, for a FFS the Met peak would occur first, and for a MFS they would approximately coincide. The second touchdown algorithm followed the same procedures but used the vertical jerk peaks of the Heel and Met markers (TDJ) (Figure 1C).

The first toe-off algorithm was also based on that proposed in a number of previous studies (Alvim et al., 2015; Leitch et al. 2011; Maiwald et al., 2009) and was given by the peak in vertical acceleration of the Toe marker within the toe-off window $\left(\mathrm{TO}_{\mathrm{A}}\right)$. The second toe-off algorithm followed the same procedure but used the vertical jerk peak of the Toe marker (TOJ) (Figure 1c).

GCT was calculated using all combinations of the touchdown and toe-off algorithms described above, giving a total of four algorithms $\left(G_{C} T_{A A}, G T_{J J}, G_{A} T_{A J}\right.$ and GCTJA; where the first letter in the subscript refers to the touchdown algorithm used and the second letter to the toe-off algorithm).

\subsection{Statistics}

The 95\% limits of agreement (Bland and Altman, 1995) were used to determine bias and random error in each kinematic method compared to the force platform gold standard. Root mean square error (RMSE) was also calculated to enable comparison with previous studies. To assess whether there was a relationship between GCT (and hence running speed) and level of agreement, Pearson's correlation coefficients were calculated between the means and difference values from the Bland-Altman analysis. 

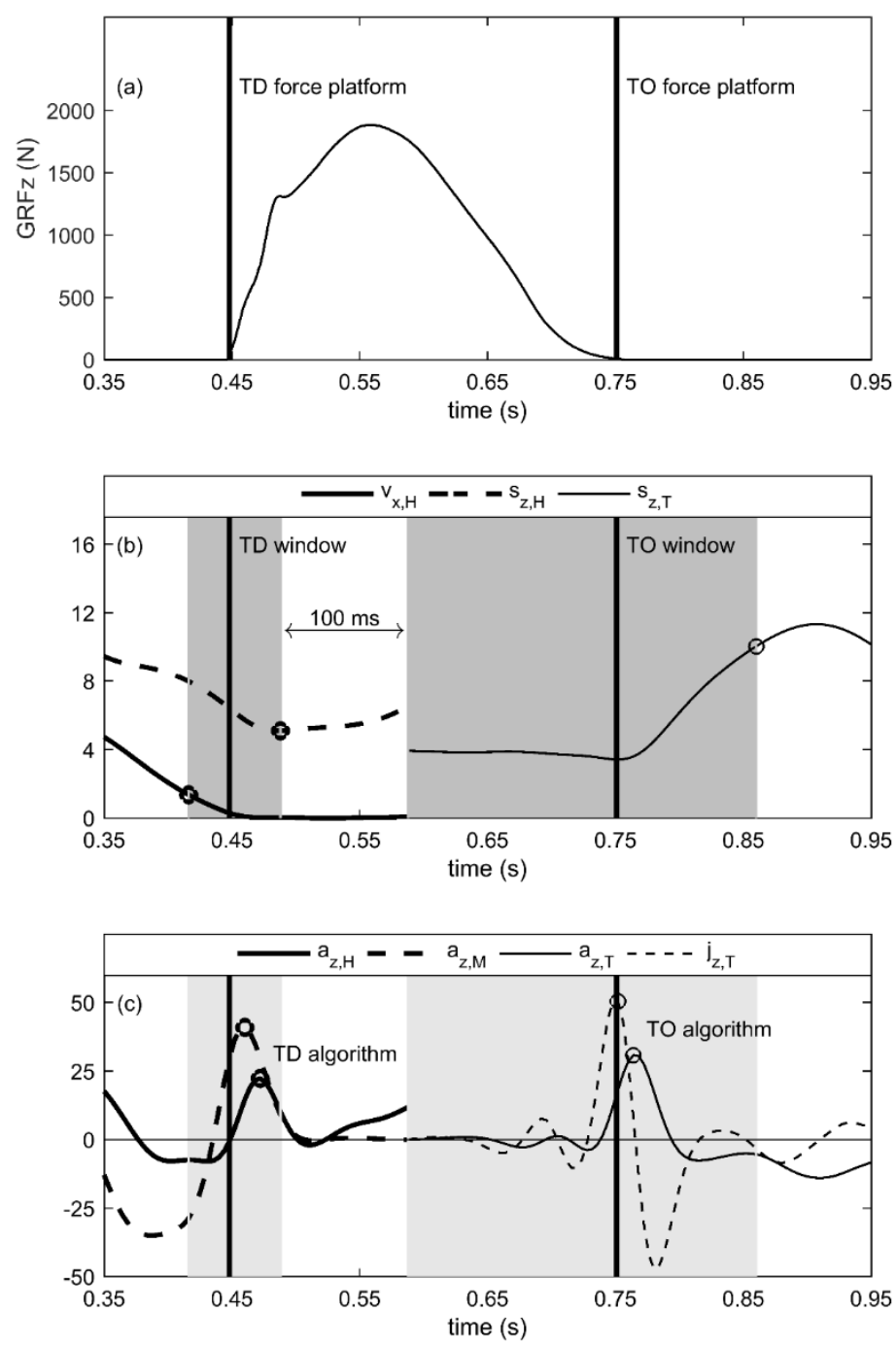

Figure 1. Illustration of the ground contact algorithms tested: (a) vertical ground reaction force (GRFz) from the force platform; (b) touchdown and toe-off search windows indicated by the shaded regions; (c) touchdown and toe-off instances estimated using kinematic algorithms. In all sub-plots the solid vertical lines indicate "gold standard" touchdown and toe-off instances. In sub-plot (b) the circles indicate the times corresponding to the start and end of the touchdown and toe-off windows. In sub-plot (c) the circles indicate the peaks in acceleration / jerk as used to estimate touchdown and toe-off instances for each kinematic algorithm. Nomenclature: GRFz is vertical ground reaction force (in N); TD is touchdown; TO is toe-off; $v x, \mathrm{H}$ is anterior-posterior velocity of the heel marker (in $\mathrm{m} \cdot \mathrm{s}-1$ ); $\mathrm{sz}, \mathrm{H}$ is vertical position of the heel marker (in cm); sz,T is vertical position of the toe marker (in $\mathrm{cm}$ ); az, $\mathrm{H}$ is vertical acceleration of the heel marker (in $\mathrm{m} \cdot \mathrm{s}-2$ ); az, $\mathrm{M}$ is vertical acceleration of the metatarsal marker (in $\mathrm{m} \cdot \mathrm{s}-2$ ); az,T is vertical acceleration of the toe marker (in $\mathrm{m} \cdot \mathrm{s}-2$ ); and jz,T is vertical jerk of the toe marker (multiplied by 0.033 and in $\mathrm{m} \cdot \mathrm{s}-3)$.

\section{RESULTS}

The results are based on a total of 538 analysed ground contacts. The overall mean and range of GCTs obtained from the force platform data were $256 \pm 56 \mathrm{~ms}$, and 139-475 ms respectively.

For identifying touchdown, the acceleration algorithm $\left(\mathrm{TD}_{\mathrm{A}}\right)$ performed better than the jerk algorithm (TDJ) (Table 1). It had a smaller offset $(+3.1 \mathrm{~ms}$ versus $-10.5 \mathrm{~ms})$ and RMSE (8.3 ms versus $12.3 \mathrm{~ms}$ ) while the $95 \%$ confidence intervals were of similar magnitude. The offsets also differed in sign; the positive value for $\mathrm{TD}_{\mathrm{A}}$ indicated that the peak occurred too late, while the negative value for TDJ indicated that the peak 
occurred too early. Across all the kinematic methods for individual events, TDJ was the only peak that systematically occurred too early.

For identifying toe-off the jerk algorithm (TOJ) performed better than the acceleration algorithm (TOA) (Table 1$)$. It had a smaller offset $(+2.1 \mathrm{~ms}$ versus +12.3 $\mathrm{ms}$ ) and RMSE (5.6 ms versus $13.1 \mathrm{~ms}$ ) while the $95 \%$ confidence intervals were of similar magnitude.

For estimating GCT the algorithm which used the acceleration peak for touchdown, and jerk peak for toe-off $\left(G_{C T} \mathrm{~J}\right)$, i.e. combining the two best performing algorithms for the individual events, performed the best (Table 1). It had the smallest offset $(-1.1 \mathrm{~ms})$, i.e. it systematically under-estimates GCT, and RMSE $(9.0 \mathrm{~ms})$, while the $95 \%$ confidence intervals were of similar magnitude across all four algorithms.

All but one of the algorithms estimating GCEs had offsets which were positively correlated with GCT (and hence running speed; $\mathrm{p}<0.01$ ) (Figure 2). $\mathrm{TO}_{\mathrm{A}}$ and TOJ exhibited large effect sizes $(r=0.52$, and $r=0.62)$, TDA exhibited a small effect size $(r=$ $0.27)$, and TD was not correlated with GCT $(r=0.03, p>0.01)$ (Cohen, 1992).

The offsets for all GCT algorithms were significantly positively correlated with GCT $(p<0.01)$ (Figure 3); GCT AA $_{\text {and }}$ GCT $_{A J}$ exhibited small effect sizes ( $r=0.11$, and $r=$ $0.20)$, and GCTJA and GCTJJ exhibited medium effect sizes ( $r=0.33$, and $r=0.42$ ) (Cohen, 1992).

Table 1. Difference data between the gold standard force platform time and each of the kinematic algorithm times for touchdown, toe-off, and ground contact time. A negative value indicates that the kinematic algorithm is early compared to the force platform (or under-predicts ground contact time) while a positive value indicates that the kinematic algorithm is late compared to the force platform (or over-predicts ground contact time).

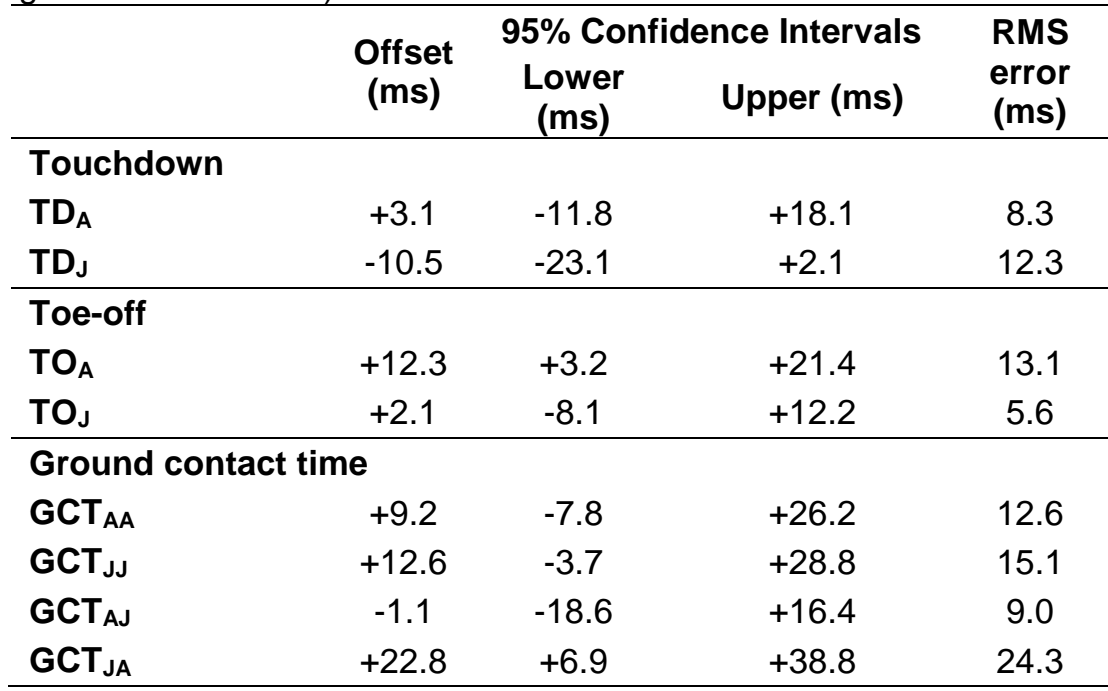

Nomenclature: $\mathrm{TD}_{\mathrm{A}}$ is touchdown based on first occurring of heel and metatarsal vertical acceleration peak; TDJ is touchdown based on first occurring of heel and metatarsal vertical jerk peak; $\mathrm{TO}_{\mathrm{A}}$ is toe-off based on toe vertical acceleration peak; TOJ is toe-off based on toe vertical jerk peak; GCT $T_{A A}$ is ground contact time based on TDA and $\mathrm{TO}_{\mathrm{A}} ; \mathrm{GCT}_{\mathrm{J} J}$ is ground contact time based on TDJ and TOJ; GCT $\mathrm{AJ}_{\mathrm{J}}$ is ground contact time based on TDA and TOJ; and GCTJA is ground contact time based on TDJ and TOA. 

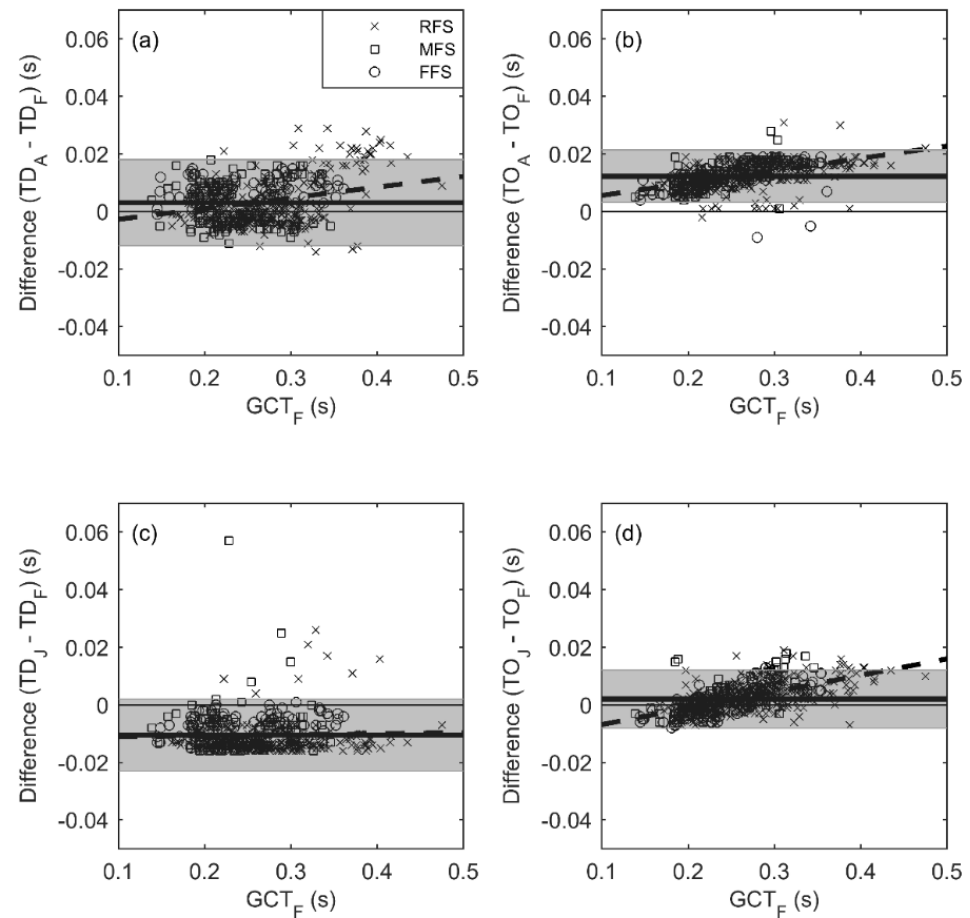

Figure 2. Plots displaying the offsets between GCEs calculated from each kinematic algorithm, and those calculated from force data, plotted against the force platform GCT. The solid horizontal line represents the mean difference (bias), the grey shaded area represents the $95 \%$ confidence intervals, and the dashed line is the best fit through the data.
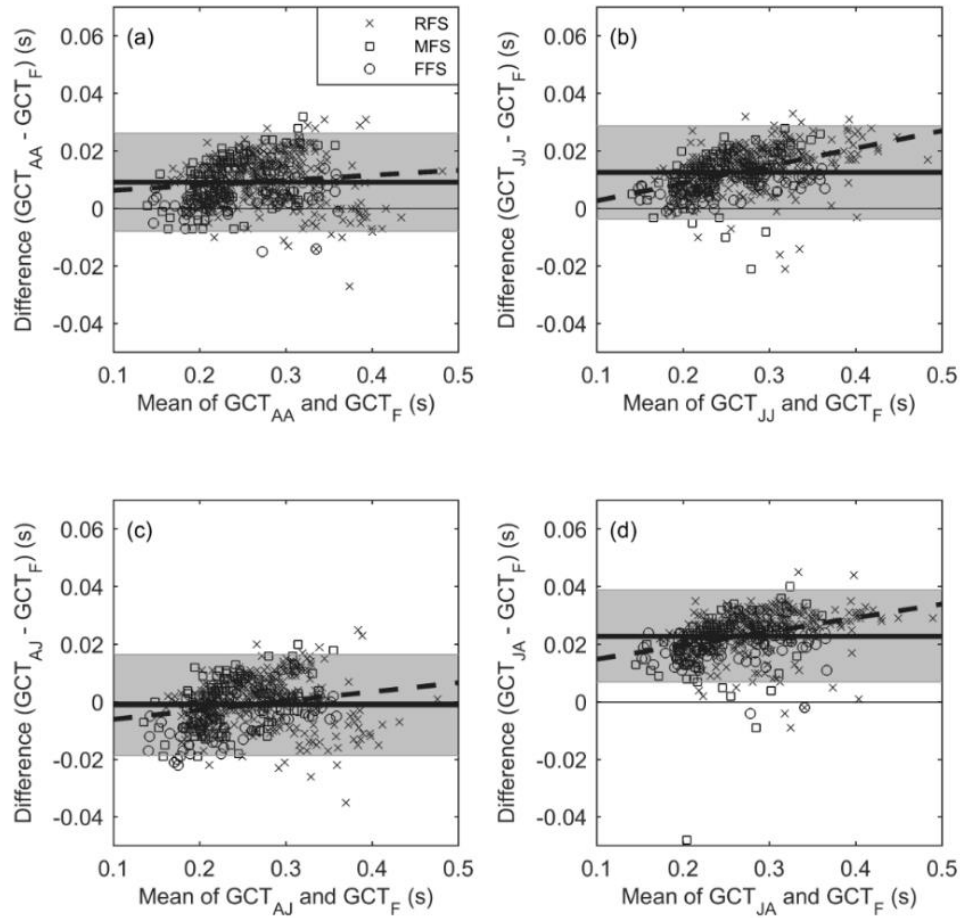

Figure 3. Bland-Altman plots displaying the level of agreement between each of the four kinematic algorithms and the force platform GCT. The solid horizontal line represents the mean difference (bias), the grey shaded area represents the $95 \%$ confidence intervals, and the dashed line is the best fit through the data. 


\section{DISCUSSION}

This is the first study to evaluate the accuracy of GCEs estimation over a range of speeds and to have included estimation of GCT and an assessment of the effect of running speed on GCT estimation within the evaluation. The intention was to determine the best algorithm for estimating GCEs from kinematic data during running at different speeds and with different footstrike types. Four algorithms were assessed; two for touchdown and two for toe-off, where in both cases one of the two was based on an algorithm previously proposed in the literature. The results suggested that using the timing of the vertical acceleration peak of either the Heel or Met marker (whichever occurred first) for touchdown (TDA), the timing of the vertical jerk peak of the Toe marker (TOJ) for toe-off, and their combination for estimating GCT (GCT AJ) provided the best estimate of GCEs across running speeds and footstrike types. The resulting RMSEs for this algorithm were $8.3 \mathrm{~ms}, 5.6 \mathrm{~ms}$, and $9.0 \mathrm{~ms}$ for touchdown, toe-off, and GCT respectively.

The use of the Heel vertical acceleration peak within the touchdown algorithm is consistent with a number of previous studies (Alvim et al., 2015; Leitch et al. 2011; Maiwald et al., 2009). However, in the current investigation the touchdown algorithm also referenced the Met vertical acceleration peak which successfully extended this algorithm to include FFS runners. Furthermore, this extended algorithm suffered no loss in accuracy (i.e. mean offset and RMS error) compared to the existing algorithms developed specifically for RFS running (Alvim et al., 2015; Hreljac \& Stergiou, 2000; Leitch et al., 2011). Approximately two thirds of the 538 footstrikes analysed in this study were RFS, with the remainder evenly split between MFS and FFS.

For identifying toe-off the Toe vertical jerk peak was found to perform better than the Toe vertical acceleration peak. A number of previous studies have proposed the latter algorithm (Alvim et al., 2015; Leitch et al. 2011; Maiwald et al., 2009); however, none of these considered an algorithm based on the Toe vertical jerk signal. The accuracy of results for the Toe vertical jerk peak algorithm are as good or better than those reported for the Toe vertical acceleration peak algorithm in these previous studies. The accuracy is also as good or better than previous algorithms based on the use of alternative lower limb based variables to determine toe-off (Fellin et al., 2010; Hreljac \& Stergiou, 2000; Smith et al., 2015).

The $\mathrm{GCT}_{\mathrm{AJ}}$ algorithm has been shown to work over a wider range of running speeds than previous studies, with similar levels of accuracy (Alvim et al., 2015; Leitch et al. 2011; Maiwald et al., 2009). It also performed as well as previous studies based on a single running speed (Smith et al., 2015) or an unspecified range of speeds (Hreljac \& Stergiou, 2000). The range of GCTs observed in this study are likely to correspond to running speeds of approximately 2-6 $\mathrm{m} \cdot \mathrm{s}^{-1}$ (Forrester and Townend, 2015).

All but one of the GCE and GCT algorithms demonstrated an effect of GCT and consequently an implied effect of running speed upon the offset of the GCE and GCT estimations (Figures $2 \& 3$ ). All offsets were positively correlated with GCT and hence negatively correlated with speed, therefore the offsets became more negative as speed increased. In all but one case (GCT $\mathrm{AJ}_{\mathrm{J}}$ ) this led to the GCT algorithms becoming more accurate at higher speeds since they had a positive mean offset (Figure 3 ) but this effect was not so pronounced in the GCE algorithms since the offsets were typically smaller (Figure 2). The best performing GCT algorithm (GCTAJ) exhibited a small effect of running speed $(r=0.20)$ on GCT offset, this translated to a range of approximately 11 $\mathrm{ms}$ ( $-5 \mathrm{~ms}$ to $6 \mathrm{~ms}$ ) over the spread of GCTs in this study. 
To conclude, when estimating GCEs during running in the absence of a force platform, an algorithm that uses the first occurring of the vertical acceleration peaks of the Heel and Met to identify touchdown and the vertical jerk peak of the Toe to identify toe-off is recommended. This algorithm requires an initial windowing step to identify reduced time windows containing touchdown and toe-off. Furthermore, this algorithm has been shown to be accurate across a range of running speeds and footstrike types.

\section{ACKNOWLEDGMENT}

This work was financially supported by MAS Holdings, Sri Lanka.

\section{CONFLICT OF INTEREST}

The authors have no conflict of interest to disclose. 


\section{REFERENCES}

Altman, A.R., Davis, I.S. A kinematic method for footstrike pattern detection in barefoot and shod runners. Gait Posture 2012; 35: 298-300.

Alvim, F., Cerqueira, L., Netto, A.D., Leite, G., Muniz, A. Comparison of five kinematicbased identification methods of foot contact events during treadmill walking and running at different speeds. J. Appl. Biomech. 2015; 31: 383-388.

Bland, J.M., Altman, D.G. Comparing methods of measurement: why plotting difference against standard method is misleading. The Lancet 1995; 346(8982): 1085-1087.

Cohen, J. A power primer. Psych. Bull. 1992; 112: 155-159.

De Witt, J.K., Determination of toe-off event time during treadmill locomotion using kinematic data. J. Biomech. 2010; 43: 3067-3069.

Fellin, R.E., Rose, W.C., Royer, T.D., Davis, I.S. Comparison of methods for kinematic identification of footstrike and toe-off during overground and treadmill running. J. Sci. Med. Sport 2010; 13: 646 -650.

Forrester, S.E., Townend, J. The effect of running velocity on footstrike angle - A curveclustering approach. Gait Posture 2015; 41: 26-32.

Hansen, A.H., Childress, D.S., Meier, M.R. A simple method for determination of gait events. J. Biomech. 2002; 35: 135-138.

Hreljac, A., Stergiou, N. Phase determination during normal running using kinematic data. Med. Biol. Eng. Comput. 2000; 38: 503-506.

Leitch, J., Stebbins, J., Paolini, G., Zavatsky, A.B. Identifying gait events without a force plate during running: a comparison of running. Gait Posture 2011; 33: 130-132.

Maiwald, C., Sterzig, T., Mayer, T.A., Milani, T.L. Detecting foot-to-ground contact from kinematic data in running. Footwear Sci. 2009; 1: 111-118.

Milner, C.E., Paquette, M.R. A kinematic method to detect foot contact during running for all foot strike patterns. J. Biomech. 2016; 48: 3502-3505.

O'Connor, C.M., Thorpe, S.K., O'Malley, M.J., Vaughan, C.L. Automatic detection of gait events using kinematic data. Gait Posture 2007; 25: 469-474.

Smith, L., Preece, S., Mason, D., Bramah, C. A comparison of kinematic algorithms to estimate gait events during overground running. Gait Posture. 2015; 41: 39-43.

Zeni, J.A., Richards, J.G., Higginson, J.S. Two simple methods for determining gait events during treadmill walking using kinematic data. Gait Posture 2008; 27: 710 714. 